\title{
Prediction of calculated future cardiovascular disease by monocyte count in an asymptomatic population
}

\author{
Deirdre F Waterhouse' \\ Ronan A Cahill ${ }^{2}$ \\ Frances Sheehan ${ }^{2}$ \\ CJ McCreery' \\ 'Department of Cardiovascular \\ Medicine, St Vincent's University \\ Hospital, Dublin, Ireland; ' 2 epartment \\ of Preventative Medicine, Blackrock \\ Clinic, Dublin, Ireland
}

Correspondence: Deirdre Waterhouse Clinical Fellow, St Vincent's University Hospital, Dublin 4, Ireland

Tel +353872368526

Fax +353 | 2832696

Email deirdrewaterhouse@ireland.com
Introduction: Although atherogenesis is clearly entwined with systemic inflammation, the risk-predictive relationship between preclinical and overt cardiovascular disease (CVD) and systemic white blood cell (WBC) subtypes remains unclear. Implication of an association would greatly facilitate cardiac risk prediction, assessment and monitoring.

Methods: 1383 asymptomatic individuals (795 men, 588 women) attending for executive health screening were examined clinically as well as with phlebotomy and exercise stress testing to determine their ten-year risk of developing overt cardiovascular disease (as estimated by both Framingham and SCORE calculations). The significance of their association with overall WBC and subtypes were determined using both univariate and multiple regression modeling.

Results: Of all WBC subtypes, monocyte count was found to have the strongest, independent relationship with overall CVD risk by backwards linear regression modeling (Framingham: $\beta=0.057 ; p=0.03$; SCORE: $\beta=0.128 ; p=<0.0005)$. Independent associations with BMI $(\beta=5.214 ; p=<0.0005)$, waist circumference $(\beta=21.866 ; p=<0.0005)$, systolic blood pressure $(\beta=10.738 ; \mathrm{p}=0.003)$, HDL cholesterol $(\beta=-0.639 ; \mathrm{p}=<0.0005)$ and triglyceride concentrations $(\beta=0.787 ; p=<0.0005)$ were also evident. Overall WBC along with neutrophils, lymphocytes and basophil subfractions were variably (but less strongly) associated with such dependents and outcome measures.

Conclusions: In conclusion, monocyte count, a simple inexpensive test, may provide useful predictive cardiovascular risk information in asymptomatic individuals to inform and guide attempts at interrupting CVD development at a preclinical stage.

Keywords: leukocyte, white cell count, monocyte, cardiovascular risk, asymptomatic population

\section{Introduction}

Inflammation plays a key role in the pathogenesis of atherosclerosis and in the development of clinically apparent cardiovascular disease (CVD) (Yarnell et al 1991; Ross 1993, 1999; Libby 1995; Falk et al 1995; Tracey 1998; Ridker 1998). Elevated levels of systemic inflammatory markers have been shown to be associated with an increased risk of symptomatic coronary heart disease (CHD) (Folsom et al 1995, 1997; Danesh et al 2000), while total white blood cell (WBC) count has itself been shown to be an independent risk factor for both CHD development (Danesh et al 1998) and CVD-related morbidity and mortality (Kannel et al 1992; Horne et al 2005). Although a role as a biomarker of cardiovascular risk has been suggested for total WBC (Grimm et al 1985; Kuller et al 1996; Pepys and Berger 2001; Pearson et al 2003), the relative ability of specific WBC subtypes to predict cardiovascular risk in asymptomatic individuals remains largely unexamined. Such an analysis may provide greater insight into the natural history of actual cardiovascular risk. While neutrophil levels have 
proven useful in reflecting overt ischemic events post-hoc, a preclinical indicator of impending atherosclerotic crisis could provide greater therapeutic opportunity.

Thus, in this prospective observational study, the predictive ability of total and specific WBC subtypes on predicted cardiovascular risk in a cohort of patients without preexisting symptomatic CVD was evaluated both alone and in comparison to conventional risk factors.

\section{Methods}

\section{Study sample}

The study population consisted of consecutive asymptomatic males and females without prior history of clinically apparent cardiovascular disease and aged between 33 and 75 years who attended an executive cardiovascular health examination in the Department of Preventative Medicine, Blackrock Clinic, Dublin, Ireland, between December 2003 and March 2005. All enrolled participants were self-referred and underwent their evaluation by a physician in an outpatient setting. The study was approved by the Ethics Committee of Blackrock Clinic.

Initially, all participants completed a detailed health questionnaire to confirm the presence or absence of symptoms of heart disease (chest pain, dyspnoea, palpitations at rest or with exercise) as well as to detail known risk factors for CVD (hypertension, hypercholesterolemia, diabetes mellitus, cigarette smoking, family history of CVD) and medication usage (aspirin, statins, antihypertensives, diabetic medication). Additionally, individuals were questioned regarding symptoms and signs suggestive of acute infection (fevers, cough, sputum production, etc).

Exclusion criteria included the presence of known heart disease (including previous myocardial infarction [MI]) or symptoms suggestive of cardiac disease, peripheral vascular disease, history of stroke or symptomatic cerebral ischemia as well as the presence of symptoms consistent with current infection. Furthermore, all those taking aspirin, statin and/or anti-hypertensive medications were excluded.

\section{Cardiovascular risk assessment}

Cardiovascular assessment was performed on all included study subjects and comprised complete physical examination and fasting phlebotomy. Cardiovascular risk was determined by use of both Framingham and SCORE calculation on all individuals.

\section{Clinical examination}

Physical examination was performed on all subjects and included sphygmomanometry $(\mathrm{mmHg})$ along with measurement of waist circumference and body mass index (BMI) calculation (calculated as weight in kilograms divided by the square of height in meters; $\mathrm{kg} / \mathrm{m}^{2}$ ).

\section{Hematological and biochemical assessment}

Early morning, blood samples for serum measurement of WBC with differential as well as glucose, total cholesterol, low-density lipoprotein cholesterol (LDL), high-density lipoprotein (HDL) and triglyceride levels were drawn from an antecubital vein of participants resting in a supine position after an overnight fasting period of a minimum of ten hours. Once drawn, all samples were put on ice and were processed within 30 minutes. Total and peripheral differential WBC counts (neutrophils, lymphocytes, monocytes and basophils) were performed using a Sysmex NE-8000 hematology analyzer (TOA Medical Electronics, Kobe, Japan). Intraassay and inter-assay coefficients of variation were $<10 \%$. Enzymatic colorimetric methods using a Cobras Intgera 800 analyser (Roche, Basle, Switzerland) were used to determine the concentrations of fasting cholesterol, triglycerides and glucose (sensitivity $<0.003 \mathrm{mmol} / 1,0.4 \mathrm{mmol} / 1,0.03 \mathrm{mmol} / \mathrm{l}$, respectively). Appropriate standardization of the assays was performed at time intervals throughout the study period in compliance with quality-control measures.

\section{Framingham heart risk score and SCORE estimation}

10 -year risk of CHD (whether fatal or nonfatal) and fatal CVD (including both coronary and cerebrovascular deaths) was calculated for all patients on the basis of the Framingham Heart Risk Score (Wilson et al 1998) and SCORE project (Conroy et al 2003) formulae respectively. Both these estimations are widely used and well validated means of ascribing genderspecific cardiovascular risk. The Framingham Risk Score, derived from the Framingham Heart Study Cohort, predicts gender-specific 10-year risk of CHD development by assigning a weighting to each individual's age, sex, smoking status, total cholesterol, high-density lipoprotein cholesterol, systolic blood pressure, and the presence of diabetes. The European SCORE is a risk stratification model developed by the European Society of Cardiology that allows direct estimation of 10 -year risk of fatal CVD at any site for use in primary prevention programs. This means of risk stratification again assigns numerical weighting to each individual's age, sex, total cholesterol, systolic blood pressure, and smoking status.

\section{Statistical analysis}

Statistical analysis was performed using the SPSS Version 11 (SPSS Inc 1989-2001, Chicago, Illinois, USA). Univariate regression models were fit for each cardiovascular risk 
parameter to examine the independent relationships between differential WBC and cardiovascular risk parameters. Log transformation of variables was performed where appropriate to correct for skew (glucose, total cholesterol, HDL, LDL, and triglycerides). Subsequently, backward multiple linear regression models were fitted in order to determine whether independent associations exist between differential WBC and cardiovascular risk, as quantified by the Framingham and SCORE risk stratification models. A relationship was considered statistically significant if $\mathrm{p}<0.05$.

\section{Results}

Of 1716 subjects (1026 men, 690 women) attending for screening over the 15-month study period, 333 were excluded by the criteria detailed above. Fifty one had either known heart disease or a history of cerebrovascular accident or had symptoms suggestive of cardiac disease, peripheral vascular disease, symptomatic cerebral ischemia or current infection and were thus excluded from further evaluation. A further 282 were taking aspirin and/or a statin and/or anti-hypertensive medication on attendance. Therefore, the study group comprised 1383 participants (795 men, 588 women) who met the inclusion criteria. The baseline characteristics for our study population and the distribution of potential risk factors for CVD are displayed in Table 1.
Univariate regression models were fitted in order to quantify the association between total and differential WBC counts and standard risk parameters for the development of CVD (see Tables $2 \mathrm{a}$ and $2 \mathrm{~b}$ ). Significant relationships between total WBC count and risk parameters for cardiovascular disease development were demonstrated with WBC found to be significantly related to BMI, waist circumference, systolic blood pressure, HDL cholesterol, and triglyceride levels. Additionally, total WBC count was associated with SCORE but not to Framingham risk estimation. Of the leuckocyte subtypes, monocyte count showed the most compelling evidence of a significant relationship with cardiovascular risk and risk factors. Significant relationships were demonstrated between monocyte count and BMI, waist circumference, systolic blood pressure, HDL cholesterol and triglyceride concentrations. Furthermore, significant relationships between monocyte count and risk of CVD development using both Framingham and SCORE cardiovascular risk prediction models were evident. Univariate regression models also showed neutrophil count to be significantly associated with BMI, HDL cholesterol, and triglyceride measurements. Neutrophil count was, in addition, significantly associated with overall risk of having a fatal cardiovascular event in the next ten years, as estimated by the SCORE cardiovascular risk prediction model. Eosinophil count demonstrated significant associations with BMI, waist circumference, HDL

Table I Baseline characteristics and distribution of potential risk factors for CVD for the study population

\begin{tabular}{|c|c|c|c|c|c|c|}
\hline \multirow{2}{*}{$\begin{array}{l}\text { Variable } \\
\text { Smoking status }\end{array}$} & \multicolumn{3}{|l|}{ Female } & \multicolumn{3}{|l|}{ Male } \\
\hline & Nonsmokers & Ex-smokers & Current smokers & Nonsmokers & Ex-smokers & Current smokers \\
\hline $\mathrm{n}=$ & 427 & 89 & 72 & 456 & 252 & 87 \\
\hline Age (yrs) & $5 \mathrm{I}( \pm 7.9)$ & $50( \pm 7.5)$ & $49( \pm 6.4)$ & $56( \pm 5.1)$ & $56( \pm 5.2)$ & $56( \pm 5.6)$ \\
\hline Waist circumference $(\mathrm{cm})$ & $80.90( \pm 11.5)$ & $81.83( \pm 10.2)$ & $81.58( \pm 10.1)$ & $94.62( \pm 10.7)$ & $95.92( \pm 10.2)$ & $94.99( \pm 10.5)$ \\
\hline BMI $\left(\mathrm{kg} / \mathrm{m}^{2}\right)$ & $24.8 I( \pm 4 . I)$ & $25.35( \pm 3.4)$ & $24.48( \pm 3.95)$ & $27.01( \pm 3.4)$ & $27.66( \pm 3.4)$ & $27.28( \pm 3.37)$ \\
\hline Systolic BP (mmHg) & I I $9.48( \pm 17.8)$ & $118.94( \pm 17.7)$ & || $7.2 \mid( \pm \mid 4.6)$ & $125.52( \pm 16.5)$ & $128.5( \pm 16.5)$ & $130.25( \pm 18.5)$ \\
\hline Diastolic BP (mmHg) & $76.83( \pm 10.3)$ & $77.92( \pm 10.6)$ & $76.28( \pm 10.08)$ & $82.01( \pm 9.3)$ & $86.73( \pm 5.19)$ & $82.72( \pm 9.49)$ \\
\hline WBC count $\left({ }^{*} 10^{9}\right.$ cells $\left./ \mathrm{L}\right)$ & $6.05( \pm 1.52)$ & $6.07( \pm 1.52)$ & $7.21( \pm 1.97)$ & $6.26( \pm 1.62)$ & $6.34( \pm 1.60)$ & $7.17( \pm 1.95)$ \\
\hline Neutrophils (*109 cells/L) & $4.63( \pm 1.23)$ & $3.46( \pm 1.13)$ & $4.36( \pm I .5 I)$ & $3.65( \pm 1.26)$ & $3.68( \pm 1.07)$ & $4.30( \pm 1.47)$ \\
\hline Lymphocytes $\left(* 10^{9}\right.$ cells/L) & $\mathrm{I} .83( \pm 0.5 \mathrm{I})$ & $1.91( \pm 0.61)$ & $2.07( \pm 0.62)$ & I.8I $( \pm 0.57)$ & $1.81( \pm 0.48)$ & $2.02( \pm 0.62)$ \\
\hline Basophils $\left(* 10^{9}\right.$ cells $\left./ L\right)$ & $0.05( \pm 0.03)$ & $0.05( \pm 0.02)$ & $0.07( \pm 0.03)$ & $0.06( \pm 0.04)$ & $0.06( \pm 0.05)$ & $0.07( \pm 0.03)$ \\
\hline Monocytes $\left(* 10^{9}\right.$ cells/L) & $0.34( \pm 0.15)$ & $0.33( \pm 0.10)$ & $0.37( \pm 0.11)$ & $0.38( \pm 0.12)$ & $0.40( \pm 0.12)$ & $0.42( \pm 0.11)$ \\
\hline Eosinophils $\left(* 10^{9}\right.$ cells/L) & $0.17( \pm 0.11)$ & $0.18( \pm 0.12)$ & $0.20( \pm 0.03)$ & $0.19( \pm 0.11)$ & $0.22( \pm 0.15)$ & $0.23( \pm 0.03)$ \\
\hline Glucose $(\mathrm{mmol} / \mathrm{dl})$ & $4.99( \pm 2.12)$ & $5.48( \pm 4.4)$ & $5.38( \pm 4.89)$ & $5.10( \pm 0.63)$ & $5.14( \pm 0.7)$ & $5.20( \pm 1.08)$ \\
\hline Triglycerides (mg/dl) & $0.99( \pm 0.55)$ & $1.08( \pm 0.67)$ & $1.14( \pm 0.60)$ & $1.43( \pm 0.99)$ & $1.67( \pm 1.53)$ & I.7I $( \pm 0.84)$ \\
\hline Total cholesterol (mg/dl) & $5.74( \pm 0.96)$ & $5.79( \pm 0.99)$ & $5.74( \pm 0.99)$ & $5.93( \pm 2.91)$ & $5.91( \pm 1.01)$ & $5.76( \pm 1.02)$ \\
\hline HDL (mmol/dl) & I.91 $( \pm 0.45)$ & $1.90( \pm 0.5)$ & $1.87( \pm 0.48)$ & $1.47( \pm 0.38)$ & $1.47( \pm 0.33)$ & $1.42( \pm 0.46)$ \\
\hline $\mathrm{LDL}(\mathrm{mmol} / \mathrm{dl})$ & $3.25( \pm 0.90)$ & $3.40( \pm 0.88)$ & $3.35( \pm 0.90)$ & $3.56( \pm 0.89)$ & $3.70( \pm 0.89)$ & $3.52( \pm 1.06)$ \\
\hline Framingham risk score & $3.64( \pm 2.7)$ & $3.73( \pm 3.15)$ & $3.85( \pm 2.76)$ & $4.67( \pm 2.1)$ & $4.90( \pm 1.96)$ & $6.77( \pm 2.66)$ \\
\hline SCORE & $0.88( \pm 1.14)$ & $0.98( \pm 1.24)$ & $0.88( \pm 0.89)$ & $3.53( \pm 2.56)$ & $3.76( \pm 2.67)$ & $7.24( \pm 6.44)$ \\
\hline
\end{tabular}

Note: Data are given as mean \pm SEM value unless otherwise stated.

Abbreviations: BMI, body mass index; BP, blood pressure;WBC, white blood cell; HDL, high density lipoprotein; LDL, low density lipoprotein. 


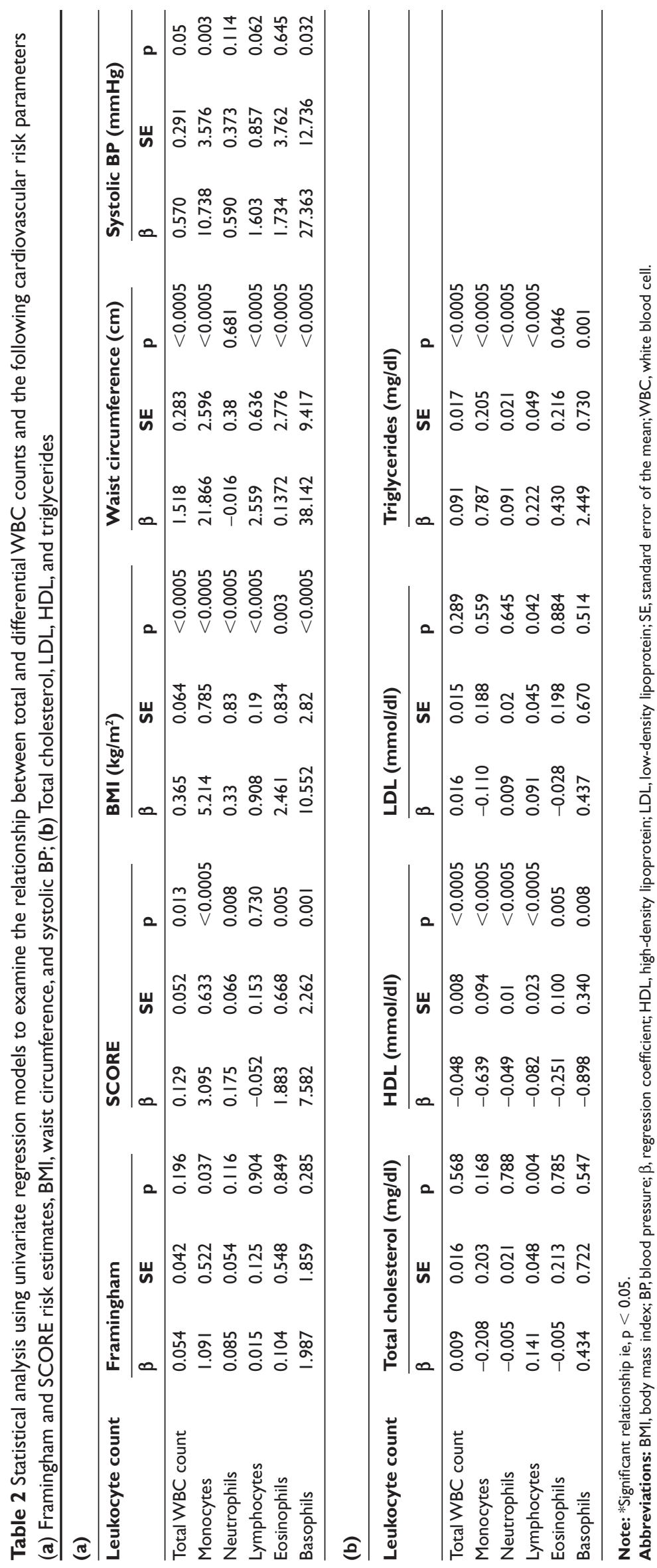


cholesterol, and triglyceride concentrations as well as with 10 year risk of having a fatal cardiovascular event, as described by SCORE. Basophil count was significantly associated with BMI, waist circumference, systolic blood pressure, HDL cholesterol, and triglyceride concentrations as well as with SCORE-estimated risk. Lymphocyte count demonstrated significant associations with BMI, waist circumference, total cholesterol, HDL cholesterol, LDL cholesterol, and triglyceride concentrations. However, despite the significant statistical significance of the relationship between lymphocyte count and risk parameters for CVD development, the association did not translate into associated increased 10 year risk of either CVD development or risk of having a fatal cardiovascular event.

On backward stepwise linear regression model analysis, monocyte count was the only WBC component found to be independently associated with both Framingham and SCORE cardiovascular risk stratification models (see Table 3a). While lymphocyte and basophil counts were also shown by this means to be independently associated with SCORE (see Table $3 \mathrm{~b}$ ), the relationship is not as compelling. Therefore, having demonstrated monocyte count to be an independent predictor of future cardiovascular risk, plots showing median monocyte count versus individual components of the FRS and SCORE were constructed (Figures 1-5). Furthermore,

Table 3 Statistical analysis using multivariable linear regression modeling to determine whether independent associations exist between (a) differential white cell count and Framingham Risk and (b) differential white cell count and SCORE.Variables entered into the stepwise regression model comprise total white cell count, neutrophils, lymphocytes, basophils, monocytes and eosinophils

(a)

Multivariable linear regression modeling examining for independent associations between differential white cell count and Framingham risk estimation.

\begin{tabular}{llll}
\hline WBC subtype & $\boldsymbol{\beta}$ & Standard error & $\mathbf{p}$ value \\
\hline Monocytes & 0.057 & 2.12 & 0.03 \\
\hline
\end{tabular}

(b)

Multivariable linear regression modeling examining for independent associations between differential white cell count and SCORE risk estimation.

\begin{tabular}{llll}
\hline WBC subtype & $\boldsymbol{\beta}$ & Standard error & p value \\
\hline Monocytes & 0.128 & 4.436 & $<0.0005$ \\
Lymphocytes & -0.082 & -2.807 & 0.005 \\
Basophils & 0.067 & 2.366 & 0.02 \\
\hline
\end{tabular}

Abbreviations: $\beta$, regression coefficient; Standard Error, standard error of regression coefficient;WBC, white blood cells.
Figure 6 demonstrates the monocyte level increment with increasing calculated Framingham and SCORE coronary risk categories. The beta co-efficient already calculated by the multivariate analyses indicated that an increment of monocyte count by 0.057 and 0.128 was associated with a $1 \%$ increase in Framingham and SCORE risk estimation, respectively.

Finally, the predictive ability of monocyte count was compared to that of BMI and waist circumference for the strength of an association with both Framingham and SCORE predictive risk of cardiovascular events (see Table 4). Additional parameters such as LDL, smoking status, age, and systolic blood pressure were not examined in this fashion as they themselves are components of both scores. Thus it can be appreciated that, while monocyte count is a predictor of future cardiovascular events, its predictive ability is exceeded by both BMI and waist circumference.

\section{Discussion}

WBC count provides a widely available and rapidly performed means of assessing systemic inflammatory status that is both well-standardized and inexpensive (Hoffman et al 2004). However despite prior studies showing WBC count to be an independent risk factor and prognostic indicator of future cardiovascular outcome that may hold true regardless of actual current clinical CVD status (Prentice et al 1982a; Zalokar 1983; Kannel et al 1992; Weiss et al 1995; Danesh et al 1998; James et al 1999; Lee et al 2001; Barron et al 2001; Haim et al 2004; Gillum et al 2005; Margolis et al 2005; Roy et al 2006), the exact clinical relevance of the association remains to be fully elucidated (Madjid et al 2004). Of WBC sub-fractions, neutrophil counts have, to date, shown the strongest association with CVD incidence and mortality (Prentice et al 1982b; Zalokar 1983; Olivares et al 1993; Kirtane et al 2004; Wheeler et al 2004; Margolis et al 2005; Gillum et al 2005) although the majority of studies have concentrated on either patients with acute symptoms or on those at highest risk of overt CVD. Recent findings that neutrophil/lymphocyte ratio provides the greatest risk prediction in patients undergoing angiographic studies for suspected CHD symptoms seem to confirm that this subtype (like C-reactive protein [CRP]) may represent either an adaptive or maladaptive response of reparative intent towards atherosclerotic plaque rupture and clinical significant ischemia (Horne et al 2005; Dragu et al 2006). Little information however is available concerning the independent predictive ability of specific WBC subtypes to predict CVD risk, independent of standard risk 
(a)

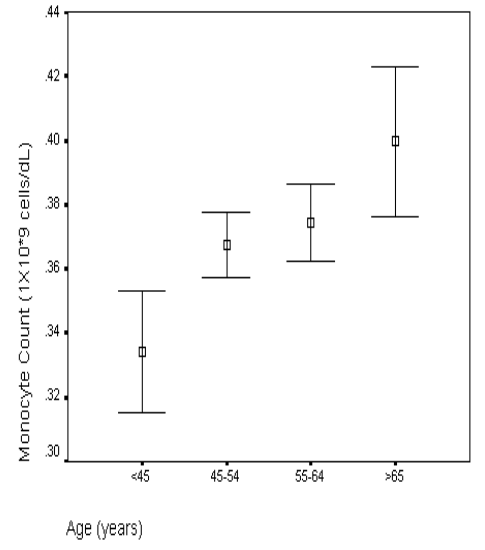

(c)

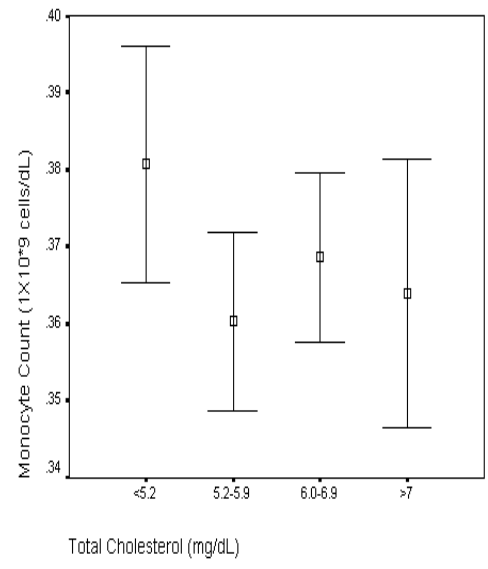

(f)

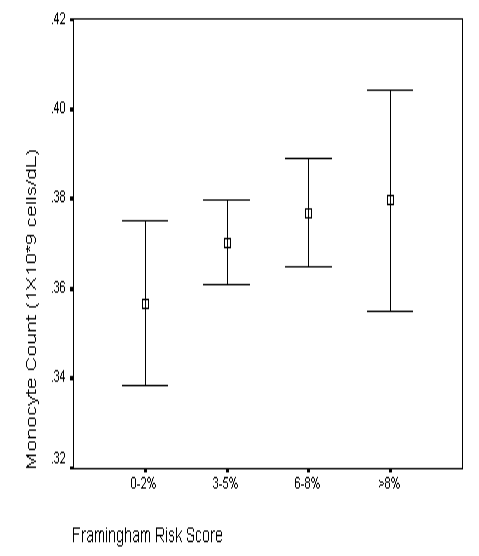

(b)

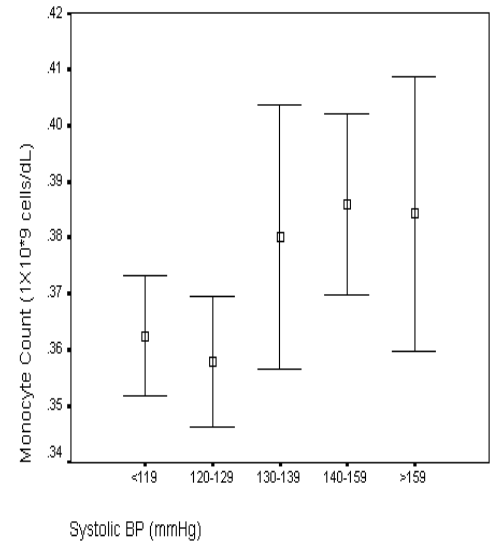

(e) (d)

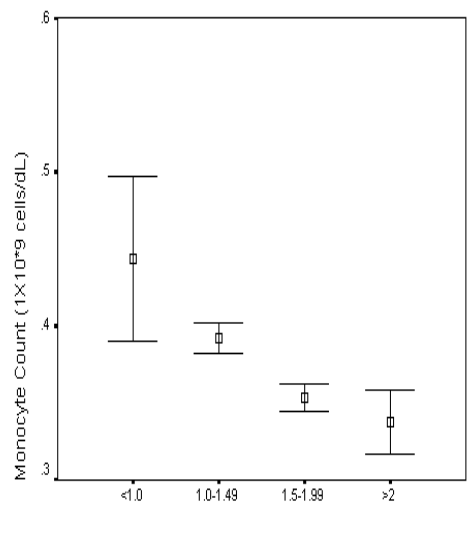

HDL Cholesterol (mmoliL)

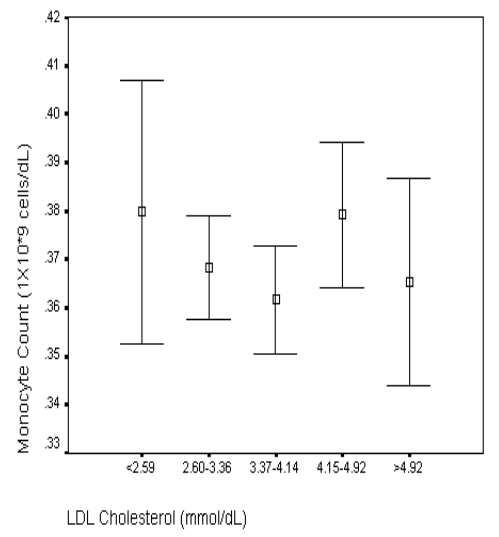

LDL Cholesterol (mmol/dL)

(g)

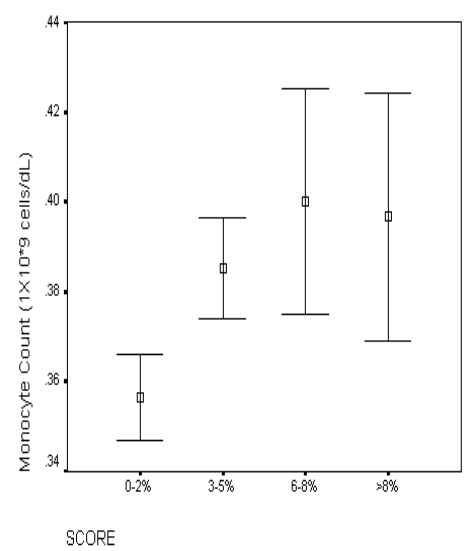

Figure I Composite figure showing relationship of median monocyte count to patient variables of (a) Age; (b) Systolic blood pressure; (c) Total cholesterol; (d) HDL cholesterol; and (e) LDL cholesterol as well as with (f) Framingham and (g) SCORE risk estimates.

Abbreviations: HDL, high-density lipoprotein; LDL, low-density lipoprotein.

factors, in individuals without overt symptoms of cardiac disease.

This prospective cohort study in an asymptomatic population demonstrates that, of all WBC subtypes, the most significant relationship with established risk parameters for CVD development exists for monocyte count and that, furthermore, this subfraction alone was independently associated with both Framingham and SCORE risk estimation. Monocytemacrophages are central mediators in the pathogenesis of atherosclerosis in both the coronary and peripheral arterial circulations (Libby 2002; Hansson 2005). Circulating monocytes become recruited to atherogenic foci where, through 


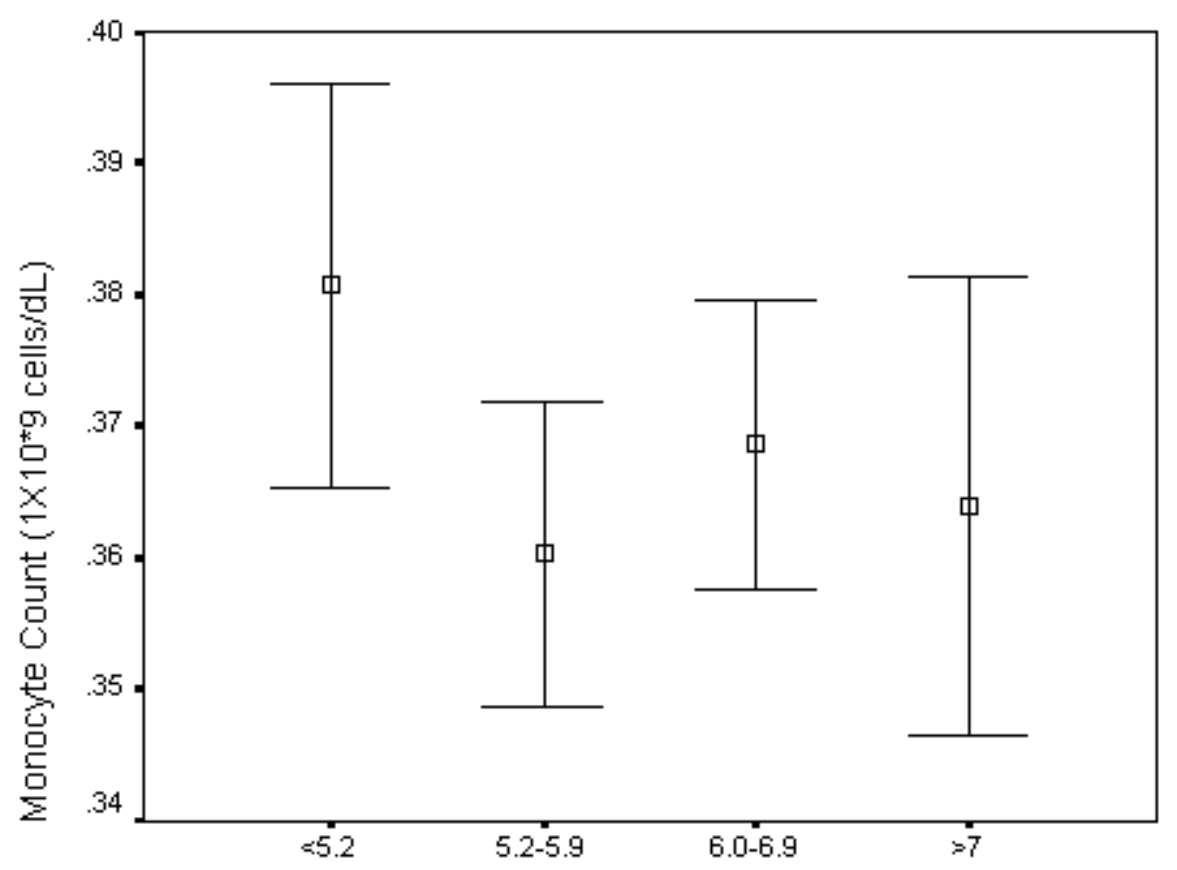

Total Cholesterol ( $\mathrm{mg} / \mathrm{dL})$

Figure 2 Comparison of median monocyte count and total cholesterol.

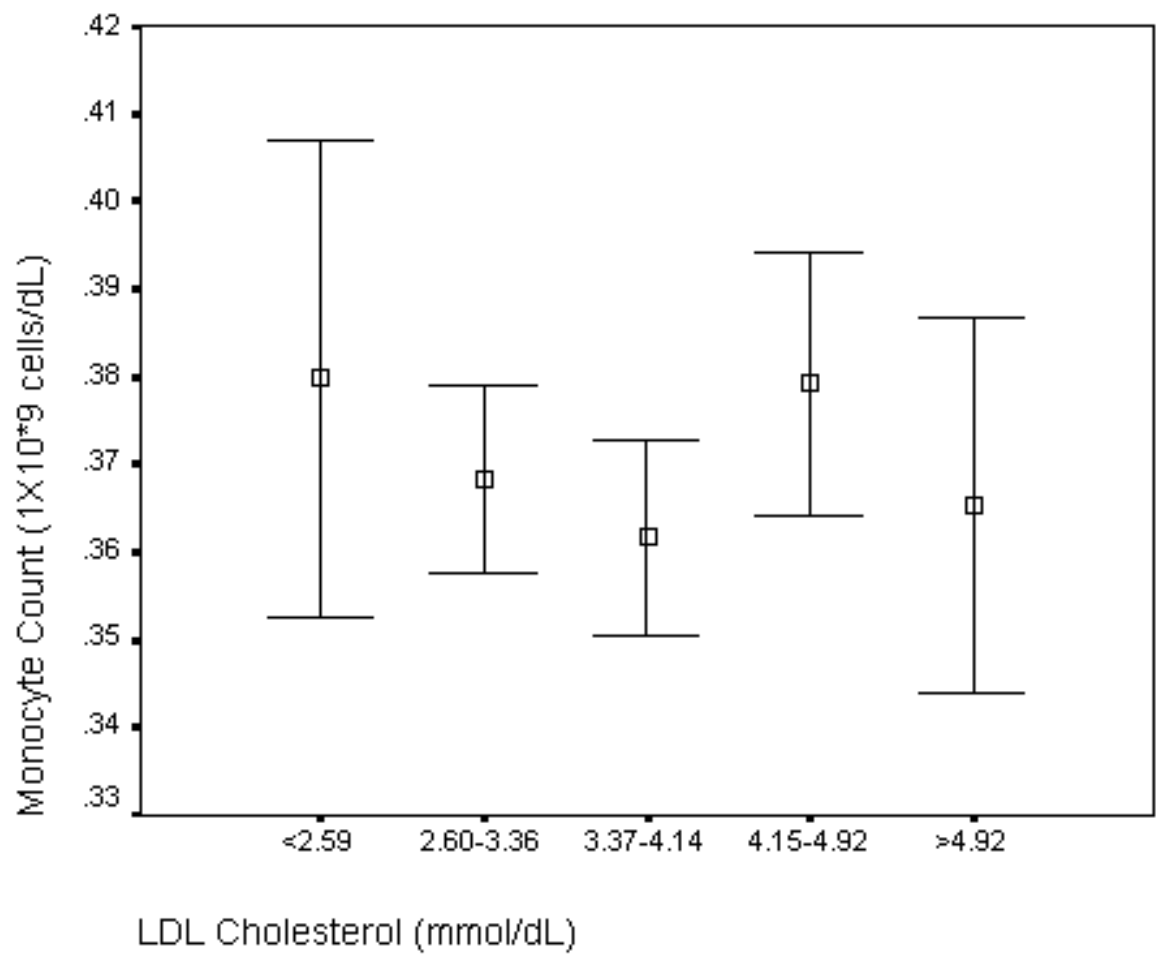

Figure 3 Comparison of median monocyte count and LDL cholesterol. Abbreviation: LDL, low-density lipoprotein. 


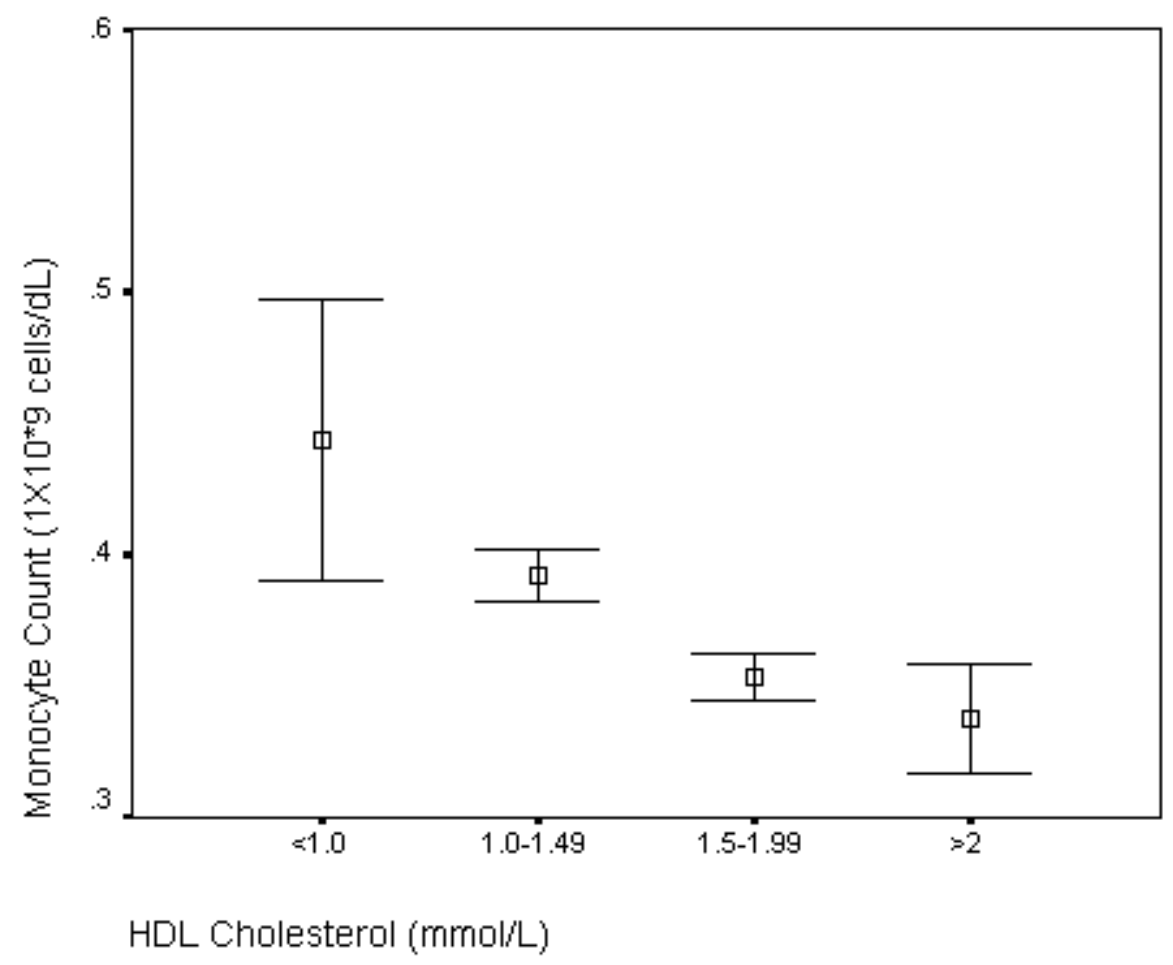

Figure 4 Comparison of median monocyte count and HDL cholesterol.

Abbreviation: HDL, high-density lipoprotein.

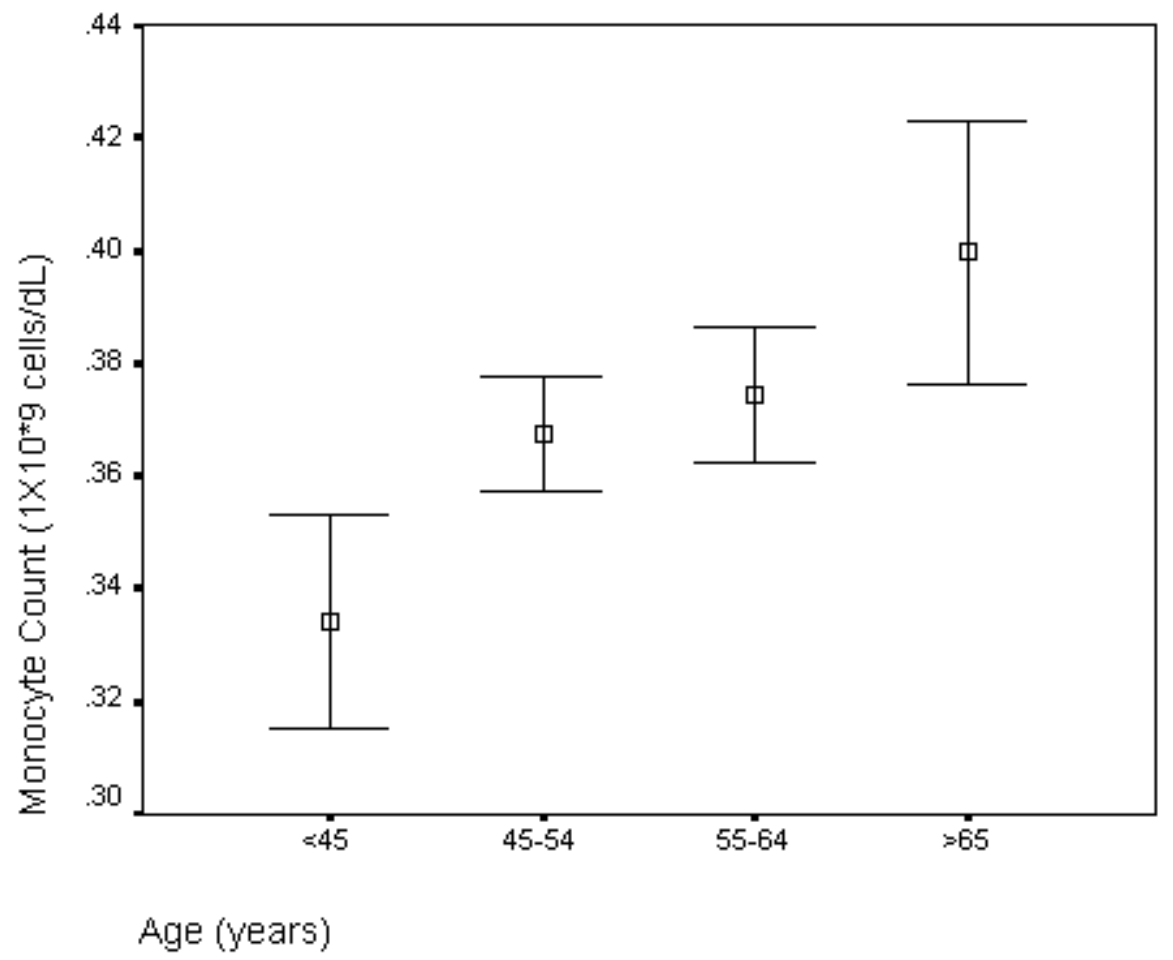

Figure $\mathbf{5}$ Comparison of median monocyte count and age. 
(a)

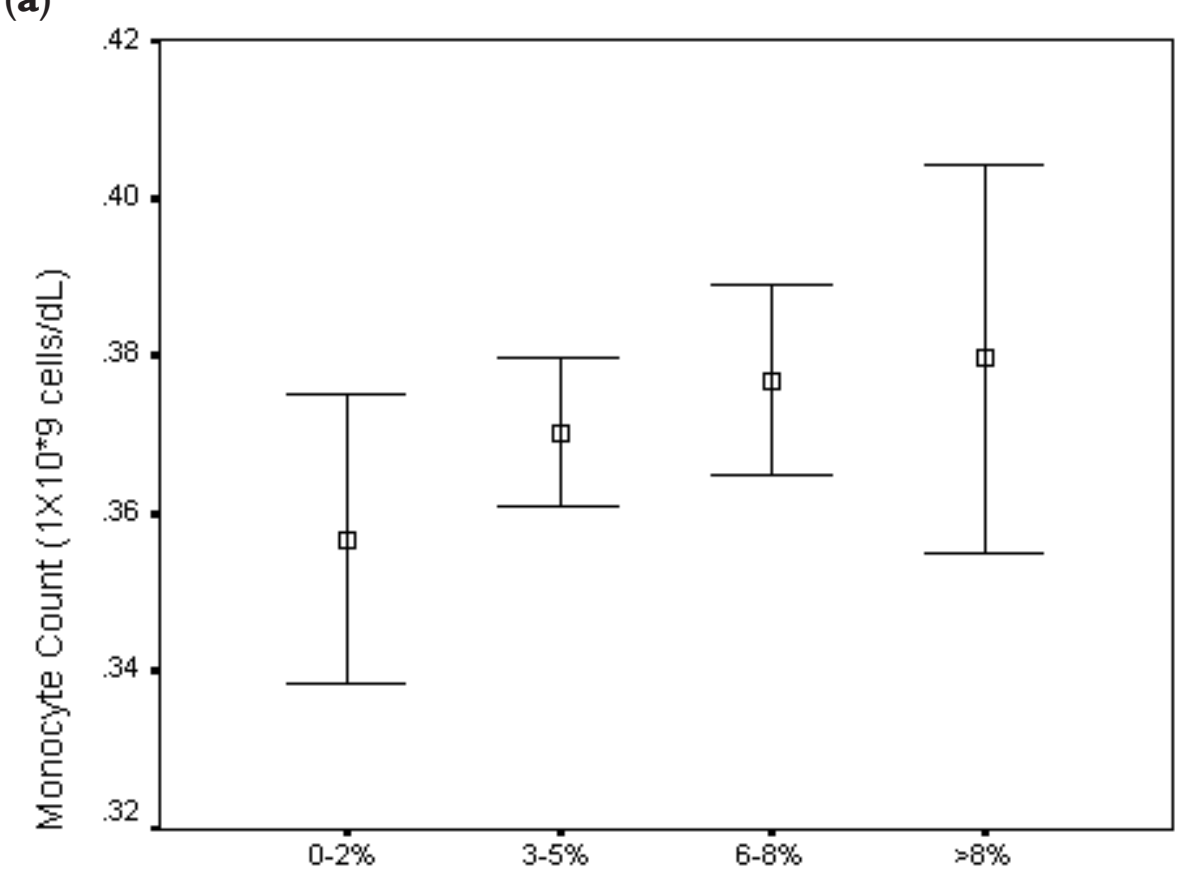

Framingham Risk Score

(b)

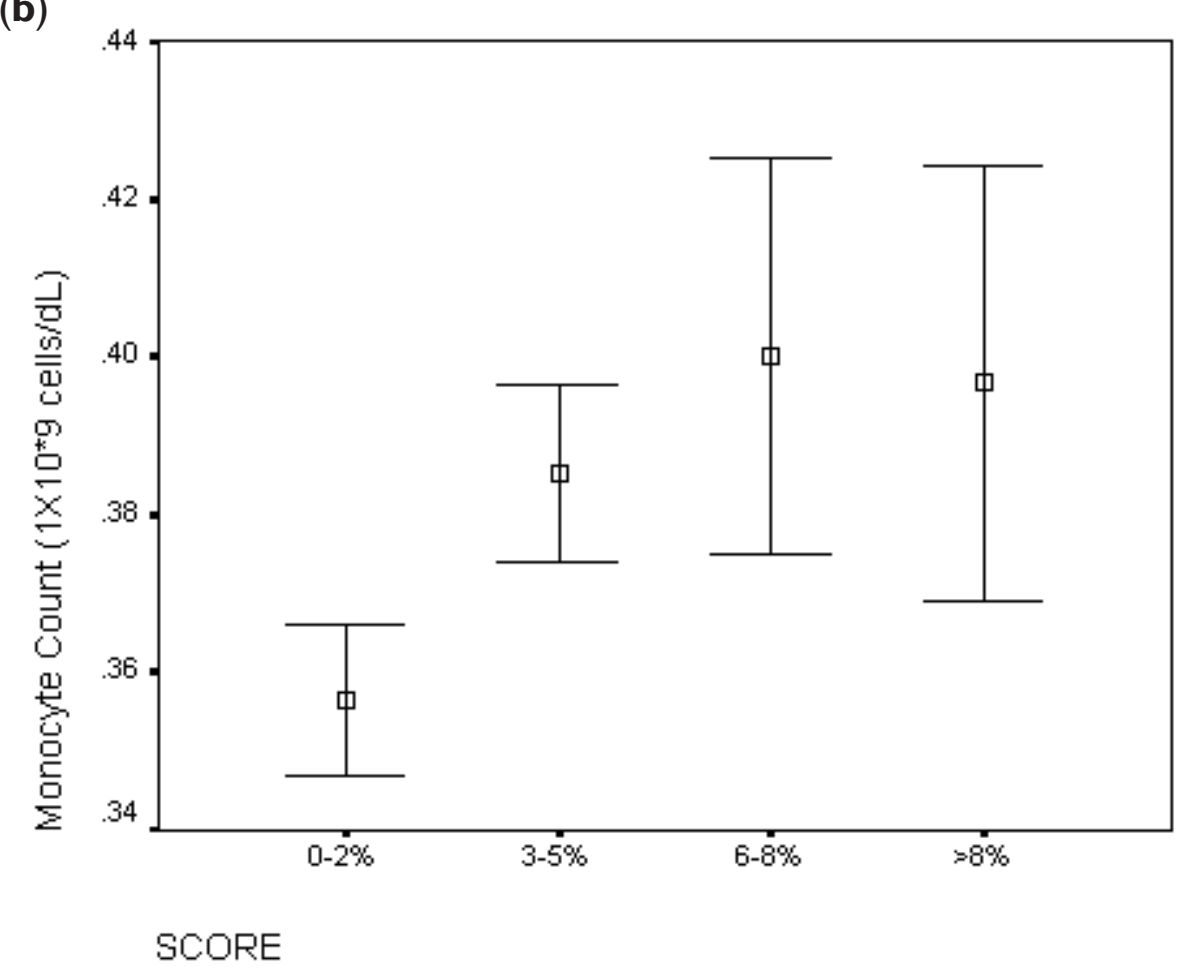

Figure 6 Comparison of (a) Framingham and (b) SCORE risk estimates with monocyte count $\left({ }^{*} 10^{9}\right.$ cells/L). 
Table 4 Statistical analysis using multivariable linear regression modeling to determine independent association between cardiovascular risk parameters and both Framingham and SCORE predictive risk estimations. Variables entered into the stepwise regression model included monocyte count, BMI, and waist circumference

\begin{tabular}{llllllll}
\hline Parameter & \multicolumn{2}{l}{ Framingham } & & & \multicolumn{2}{l}{ SCORE } \\
\cline { 2 - 3 } \cline { 6 - 8 } & $\beta$ & t value & P value & & $\beta$ & t value & P value \\
\hline Monocytes & 0.014 & 0.528 & 0.598 & & 0.064 & 2.474 & 0.013 \\
BMI & -0.073 & -1.734 & 0.083 & & -0.181 & -4.491 & $<0.0005$ \\
Waist circumference & 0.252 & 5.954 & $<0.0005$ & & 0.436 & 10.709 & $<0.0005$ \\
\hline
\end{tabular}

Abbreviations: $\beta$, Beta coeficient; $B M I$, body mass index; $t$ value, $t$ value for beta coefficient.

differentiation into macrophages, they establish a persistent cellular reaction that underlies disease progression. However, more crucial may be their association with atherosclerotic plaque instability and rupture- the precedent events of arterial thrombosis and occlusion that portend clinically significant ischemia (Falk 2006). Although it is somewhat surprising that few studies to date have directly assessed the association of circulating monocytes levels with clinically relevant cardiovascular end-points, the findings of this study are supported by the conclusions of Nasir and colleagues (2005) who also determined a significant association between monocyte count and atherosclerotic disease in patients without known cardiovascular disease albeit in the peripheral arterial circulation. Furthermore, our findings are particularly intriguing given the recent demonstration by Swirski and colleagues (2007) that certain monocyte subsets dominate hypercholesterolemia-associated monocytosis and give rise specifically to macrophages in atheromata. Finally, the utility of this WBC subtype in risk assessment seems particularly appealing given that it less inherently susceptible to fluctuations due to superimposed acute inflammatory conditions or infections than are overall WBC or neutrophil subfractions or indeed other acute phase reactants such as CRP.

As does any clinical investigation, however, this prospective, observational study has potential limitations. Although a single WBC measurement was found to be significantly associated with currently utilized estimates of future cardiovascular risk; multiple measurements over time and changes in those measurements may provide a more accurate mechanism for predicting future CVD and mortality. If this is so however, the association found by this study would represent an underestimation of the actual relationship between monocyte count and CVD, and, thus, our conclusions may in fact be conservative. Additionally, the Framingham Heart Study may not apply to countries with low risk of heart disease, and there has been some concern over its use when applied to the diabetic population, tending to underestimate an individual's probability of progressing to CHD (McEwan et al 2004).
However, despite evidence that risk estimates based on Framingham generalize well to the population of the Republic of Ireland (Haq et al 1999), and is thus applicable to our study population, there is concern that this risk estimate (and indeed others) (Empana et al 2003) may overestimate absolute risk in European populations (Menotti et al 2000; Pyorala 2000; Thomsen et al 2002; Hense et al 2003). Therefore, to ensure an accurate and reliable estimate of absolute CVD risk in our study population, we additionally stratified each participant according to SCORE criteria, a risk stratification model that has specifically validated in European countries with similar levels of risk as Ireland and previously shown to be useful in ascribing risk in asymptomatic populations similar to our own (Aktas et al 2004). Although the validity of such scores has been questioned by some (Topol and Lauer 2003), they have nonetheless been integrated into primary cardiovascular disease prevention guidelines.

The findings of this study therefore suggest that monocyte count, a simple inexpensive test, may provide important risk information to aid prediction of future CVD development in disease free adults and so may help guide therapeutic intervention aimed at its interruption.

\section{Disclosure}

No conflicts of interest exist with regard to this manuscript

\section{References}

Aktas MK, Ozduran V, Pothier CE, et al. 2004. Global risk scores and exercise testing for predicting all-cause mortality in a preventive medicine program. JAMA, 292:1462-8.

Barron HV, Harr SD, Radford MJ, et al. 2001. The association between white blood cell count and acute myocardial infarction mortality in patients $\geq$ 65 years of age: findings from the cooperative cardiovascular project. J Am Coll Cardiol, 38:1654-61.

Conroy RM, Pyorala K, Fitzgerald AP, et al. 2003. Estimation of ten-year risk of fatal cardiovascular disease in Europe: the SCORE project. Eur Heart J, 24:987-1003.

Danesh J, Collins R, Appleby P, et al. 1998. Association of fibrinogen, C-reactive protein, albumin, or leukocyte count with coronary heart disease: meta-analyses of prospective studies. JAMA, 279:1477-82.

Danesh J, Collins R, Peto R. 2000. Lipoprotein(a) and coronary heart disease. Meta-analysis of prospective studies. Circulation, 102:1082-5. 
Dragu R, Huri S, Zuckerman R, et al. 2006. Predictive value of white blood cell subtypes for long-term outcome following myocardial infarction. Atherosclerosis, Dec 13; [Epub ahead of print].

Empana JP, Ducimetiere P, Arveiler D, et al. 2003. Are the Framingham and PROCAM coronary heart disease risk functions applicable to different European populations? The PRIME Study. Eur Heart $J$, 24:1903-11.

Falk E, Shah PK, Fuster V. 1995. Coronary plaque disruption. Circulation, 92:657-71.

Falk E. 2006. Pathogenesis of atherosclerosis. J Am Coll Cardiol, 47(8 Suppl):C7-12.

Folsom AR, Ma J, Eckfeldt JH, et al. 1995. Low serum albumin. Association with diabetes mellitus and other cardiovascular risk factors but not with prevalent cardiovascular disease or carotid artery intima-media thickness. The Atherosclerosis Risk in Communities (ARIC) Study Investigators. Ann Epidemiol, 5:186-91.

Folsom AR, Wu KK, Rosamond WD, et al. 1997. Prospective study of hemostatic factors and incidence of coronary heart disease: the Atherosclerosis Risk in Communities (ARIC) Study. Circulation, 96:1102-8.

Gillum RF, Mussolino ME, Madans JH. 2005. Counts of neutrophils, lymphocytes, and monocytes, cause-specific mortality and coronary heart disease: the NHANES-I epidemiologic follow-up study. Ann Epidemiol, 15:266-71.

Grimm RH Jr, Neaton JD, Ludwig W. 1985. Prognostic importance of the white blood cell count for coronary, cancer, and all-cause mortality. JAMA, 254:1932-7.

Haim M, Boyko V, Goldbourt U, et al. 2004. Predictive value of elevated white blood cell count in patients with preexisting coronary heart disease: the Bezafibrate Infarction Prevention Study. Arch Intern Med, 164:433-9.

Hansson GK. 2005. Inflammation, atherosclerosis, and coronary artery disease. $N$ Engl J Med, 352:1685-95.

Haq IU, Ramsay LE, Yeo WW, et al. 1999. Is the Framingham risk function valid for northern European populations? A comparison of methods for estimating absolute coronary risk in high risk men. Heart, 81:40-6.

Hense HW, Schulte H, Lowel H, et al. 2003. Framingham risk function overestimates risk of coronary heart disease in men and women from Germany - results from the MONICA Augsburg and the PROCAM cohorts. Eur Heart J, 24:937-45.

Hoffman M, Blum A, Baruch R, et al. 2004. Leukocytes and coronary heart disease. Atherosclerosis, 172:1-6.

Horne BD, Anderson JL, John JM, et al. 2005. Intermountain Heart Collaborative Study Group: Which white blood cell subtypes predict increased cardiovascular risk? J Am Coll Cardiol, 45:1638-43.

James AL, Knuiman MW, Divitini ML, et al. 1999. Associations between white blood cell count, lung function, respiratory illness and mortality: the Busselton Health Study. Eur Respir J, 13:1115-19.

Kannel WB, Anderson K, Wilson PW. 1992. White blood cell count and cardiovascular disease: insights from the Framingham Study. JAMA, 267:1253-6.

Kirtane AJ, Bui A, Murphy SA, et al. 2004. Association of peripheral neutrophilia with adverse angiographic outcomes in ST-elevation myocardial infarction. Am J Cardiol, 93:532-6.

Kuller LH, Tracy RP, Shaten J, et al. 1996. Relation of C-reactive protein and coronary heart disease in the MRFIT nested case-control study. Multiple Risk Factor Intervention Trial. Am J Epidemiol, 144:537-47.

Lee CD, Folsom AR, Nieto FJ, et al. 2001. White blood cell count and incidence of coronary heart disease and ischemic stroke and mortality from cardiovascular disease in African-American and White men and women: atherosclerosis risk in communities study. Am J Epidemiol, 154:758-64.

Libby P. 1995. Molecular bases of the acute coronary syndromes. Circulation, 91:2844-50.

Libby P. 2002. Inflammation in atherosclerosis. Nature, 420:868-74.

Madjid M, Awan I, Willerson JT, et al. 2004. Leukocyte count and coronary heart disease: implications for risk assessment. $\mathrm{J} \mathrm{Am} \mathrm{Coll} \mathrm{Cardiol,}$ 44:1945-56.
Margolis KL, Manson JE, Greenland P, et al. 2005. Leukocyte count as a predictor of cardiovascular events and mortality in postmenopausal women: the Women's Health Initiative Observational Study. Arch Intern Med, 165:500-8.

Menotti A, Puddu PE, Lanti M. 2000. Comparison of the Framingham risk function-based coronary chart with risk function from an Italian population study. Eur Heart J, 21:365-70.

McEwan P, Williams JE, Griffiths JD, et al. 2004. Evaluating the performance of the Framingham risk equations in a population with diabetes. Diabet Med, 21:318-23.

Nasir K, Guallar E, Navas-Acien A, et al. 2005. Relationship of monocyte count and peripheral arterial disease: results from the National Health and Nutrition Examination Survey 1999-2002. Arterioscler Thromb Vasc Biol, 25:1966-71.

Olivares R, Ducimetiere P, Claude JR. 1993. Monocyte count: a risk factor for coronary heart disease? Am J Epidemiol, 137:49-53.

Pearson TA, Mensah GA, Alexander RW, et al. 2003. Markers of inflammation and cardiovascular disease: application to clinical and public health practice: a statement for healthcare professionals from the Centers for Disease Control and Prevention and the American Heart Association. Circulation, 107:499-511.

Pepys MB, Berger A. 2001. The renaissance of $\mathrm{C}$ reactive protein. $B M J$, 322:4-5.

Prentice RL, Szatrowski TP, Kato H, et al. 1982a. Leukocyte counts and cerebrovascular disease. J Chronic Dis, 35:703-1.

Prentice RL, Szatrowski TP, Fujikura T, et al. 1982b. Leukocyte counts and coronary heart disease in a Japanese cohort. Am J Epidemiol, 116:496-509.

Pyorala K. 2000. Assessment of coronary heart disease risk in populations with different levels of risk. Eur Heart J, 21:348-50.

Ridker PM. 1998. C-reactive protein and risks of future myocardial infarction and thrombotic stroke. Eur Heart J, 19:1-3.

Ross R. 1993. The pathogenesis of atherosclerosis: a perspective for the 1990s. Nature, 362:801-9.

Ross R. 1999. Atherosclerosis - an inflammatory disease. N Engl J Med, 340:115-26.

Roy D, Quiles J, Avanzas P, et al. 2006. A comparative study of markers of inflammation for the assessment of cardiovascular risk in patients presenting to the emergency department with acute chest pain suggestive of acute coronary syndrome. Int J Cardiol, 109:317-21.

Swirski FK, Libby P, Aikawa E, et al. 2007. Ly-6Chi monocytes dominate hypercholesterolemia-associated monocytosis and give rise to macrophages in atheromata. $J$ Clin Invest, 117:195-205.

Thomsen TF, McGee D, Davidsen M, et al. 2002. A cross-validation of risk-scores for coronary heart disease mortality based on data from the Glostrup Population Studies and Framingham Heart Study. Int $J$ Epidemiol, 31:817-22.

Topol EJ, Lauer MS. 2003. The rudimentary phase of personalised medicine: coronary risk scores. Lancet, 362:1776-7.

Tracey RP. 1998. Inflammation in cardiovascular disease: cart, horse, or both? Circulation, 97:2000-2.

Weiss ST, Segal MR, Sparrow D, et al. 1995. Relation of FEV1 and peripheral blood leukocyte count to total mortality. The Normative Aging Study. Am J Epidemiol, 142:493-8; Discussion, 499-503.

Wheeler JG, Mussolino ME, Gillum RF, et al. 2004. Associations between differential leucocyte count and incident coronary heart disease: 1764 incident cases from seven prospective studies of 30,374 individuals. Eur Heart J, 25:1287-92.

Wilson PW, D'Agostino RB. Levy D, et al. 1998. Prediction of coronary heart disease using risk factor categories. Circulation, 97:1837-47.

Yarnell JW, Baker IA, Sweetnam PM, et al. 1991. Fibrinogen, viscosity, and white blood cell count are major risk factors for ischemic heart disease. The Caerphilly and Speedwell collaborative heart disease studies. Circulation, 83:836-44.

Zalokar JB. 1983. Leukocyte counts and coronary heart disease in a Japanese cohort. Am J Epidemiol, 118:611-12. 
